\title{
Categorical properties of racks
}

Hatice Gulsun Akay and Ibrahim Ilker Akca

Eskisehir Osmangazi University, Department of Mathematics and Computer Science, Eskisehir, Turkey

Received: 13 November 2017, Accepted: 11 January 2018

Published online: 21 February 2018.

Abstract: In this paper, we give some categorical objects of racks such as product, pullback and equalizer objects.

Keywords: Rack, pullback, equalizer.

\section{Introduction}

A rack [3] is a set with a non-associative binary operation satisfying two rack conditions. The theory of racks is connected to the group theory. This relation leads to the functor Conj: Grp $\rightarrow$ Rack between the categories of racks and of groups which admits a left adjoint functor As: Rack $\rightarrow \mathbf{G r p}$; see [4], [7] for more details.

The earliest work on racks is due to Conway and Wraith [3] which is inspired by the conjugacy operation in a group and focuses in the special case of racks, called quandles; but they also were aware of the generalization. In the literature, racks are also called "automorphic sets" [2], "crystals" [8] and "(left) distributive quasigroups" [10].

In this study, we firstly recall the definitions and some examples for racks. Most of them appear in [7]. Afterwards, we give some categorical properties of racks which are the constructions of product, pullback and equalizer objects. These categorical objects are defined by the universal property diagrams in [1], [9] and examined for more specific categories such as category of crossed modules of racks and (modified) categories of interest in [5], [6].

\section{Racks}

We recall some notions from [7] which will be used in sequel.

Definition 1. A rack $R$ is a set with a binary operation satisfying:

(R1) For all $a, b \in R$, there exists a unique $c \in R$ such that:

$$
c \triangleleft a=b,
$$

(R2) For all $a, b, c \in R$, we have:

$$
(a \triangleleft b) \triangleleft c=(a \triangleleft c) \triangleleft(b \triangleleft c) .
$$

A rack which aditionally satisfies the idempotency condition:

$$
r \triangleleft r=r
$$

is called a "quandle" (for all $r \in R$ ). 
Definition 2. A "pointed" rack $R$ is a rack equipped with a fixed element $1 \in R$ such that (for all $r \in R$ ):

$$
1 \triangleleft r=1 \quad \text { and } \quad r \triangleleft 1=r .
$$

Remark. We only work with the pointed racks in the rest.

Definition 3. Let $R$ and $S$ be two (pointed) racks. A rack morphism is a map:

$$
f: R \rightarrow S
$$

such that:

$$
f\left(r \triangleleft r^{\prime}\right)=f(r) \triangleleft f\left(r^{\prime}\right) \quad(\text { and } f(1)=1)
$$

for all $r, r^{\prime} \in R$.

Thus we get the category of (pointed) racks denoted by Rack.

Some examples of racks are:

(1) The trivial rack $T_{n}$ of order $n$ is the set $\{0,1,2, \ldots, n-1\}$ with the rack operation (for all $x, y \in T_{n}$ ):

$$
x \triangleleft y=x .
$$

The infinitive trivial rack $T_{\infty}$ is the set $\mathbb{Z}$ equipped with the same operation.

(2) The dihedral rack $D_{n}$ is the set $\{0,1,2, \ldots, n-1\}$ with the rack operation:

$$
x \triangleleft y=2 y-x \bmod n
$$

for all $x, y \in D_{n}$ and the infinitive dihedral rack $D_{\infty}$ is the set $\mathbb{Z}$ equipped with:

$$
x \triangleleft y=2 y-x
$$

for all $x, y \in \mathbb{Z}$.

(3) The cyclic rack $C_{n}$ of order $n$ is the set $\{0,1,2, \ldots, n-1\}$ with the rack operation:

$$
x \triangleleft y=x+1 \quad \bmod n
$$

for all $x, y \in C_{n}$, while the infinitive cyclic rack is the set $\mathbb{Z}$ equipped with:

$$
x \triangleleft y=x+1
$$

for all $x, y \in \mathbb{Z}$.

(4) Given a group $G$, we may define a rack structure on $G$ by setting (for all $g, h \in G$ ):

$$
g \triangleleft h=h^{-1} g h .
$$

This rack is called the "conjugation" rack of $G$ and denoted by ConjG. This construction provides a functor:

$$
\text { Conj : Grp } \rightarrow \text { Rack }
$$

(5) We may define a different rack structure on $G$ by setting (for all $g, h \in G$ ):

$$
g \triangleleft h=h g^{-1} h .
$$


that is called "core" rack. However this construction is not functorial.

(6) Let $P$ and $R$ be two racks, then the cartesian product:

$$
P \times R=\{(p, r) \mid p \in P, r \in R\}
$$

has a rack structure with:

$$
(p, r) \triangleleft\left(p^{\prime}, r^{\prime}\right)=\left(p \triangleleft p^{\prime}, r \triangleleft r^{\prime}\right)
$$

for all $(p, r),\left(p^{\prime}, r^{\prime}\right) \in P \times R$.

Definition 4. Let $R$ be a rack and $X$ be a set. We say that $X$ is an $R$-set when there are bijections $(\cdot r): X \rightarrow X$ for all $r \in R$ such that:

$$
(x \cdot r) \cdot r^{\prime}=\left(x \cdot r^{\prime}\right) \cdot\left(r \triangleleft r^{\prime}\right)
$$

for all $x \in X$ and $r, r^{\prime} \in R$.

Definition 5. Let $R, S$ be two racks. We say that $S$ acts on $R$ by automorphisms when there is a (right) rack action of $S$ on $R$ and:

$$
\left(r \triangleleft r^{\prime}\right) \cdot s=(r \cdot s) \triangleleft\left(r^{\prime} \cdot s\right)
$$

for all $s \in S$ and $r, r^{\prime} \in R$.

The following notion is likely to be semi-direct product of groups:

Definition 6. If there exists a (right) rack action of $R$ on $S$, the "hemi-semi-direct product" $S \rtimes R \subset S \times R$ is the rack defined by the rack operation:

$$
(s, r) \triangleleft\left(s^{\prime}, r^{\prime}\right)=\left(s \cdot r^{\prime}, r \triangleleft r^{\prime}\right)
$$

for all $(s, r),\left(s^{\prime}, r^{\prime}\right) \in S \rtimes R$.

Definition 7. For a given rack $R$, a non empty subset $S \subseteq R$ is called a subrack if $s \triangleleft s^{\prime} \in S$ for all $s, s^{\prime} \in S$.

\section{Categorical properties of racks}

In this section we give the constructions of product, pullback and equalizer objects for the category of racks.

Theorem 1. The category of racks has products.

Proof. Let $P$ and $R$ be two racks. Define:

$$
P \times R=\{(p, r) \mid p \in P, r \in R\}
$$

We already know that $P \times R$ is a rack. Also it is easy to verify that the projection maps $p_{1}: P \times R \rightarrow P$ and $p_{2}: P \times R \rightarrow R$ are rack morphisms.

Now we will check the universal property. Let $T$ be any rack and $\alpha: T \rightarrow P, \beta: T \rightarrow R$ be two rack morphisms. Then we need to prove that there exists a unique rack morphism:

$$
\varphi: T \rightarrow P \times R
$$


such that makes following diagram commutes:

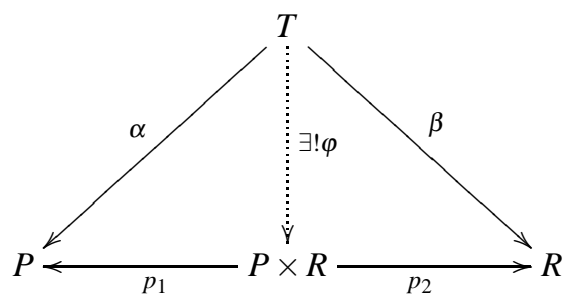

Define:

$$
\begin{aligned}
\varphi: T & \rightarrow P \times R \\
t & \mapsto \varphi(t)=(\alpha(t), \beta(t)) .
\end{aligned}
$$

$\varphi$ is a rack morphism since:

$$
\begin{aligned}
\varphi\left(t \triangleleft t^{\prime}\right) & =\left(\alpha\left(t \triangleleft t^{\prime}\right), \beta\left(t \triangleleft t^{\prime}\right)\right) \\
& =\left(\alpha(t) \triangleleft \alpha\left(t^{\prime}\right), \beta(t) \triangleleft \beta\left(t^{\prime}\right)\right) \\
& =(\alpha(t), \beta(t)) \triangleleft\left(\alpha\left(t^{\prime}\right), \beta\left(t^{\prime}\right)\right) \\
& =\varphi(t) \triangleleft \varphi\left(t^{\prime}\right)
\end{aligned}
$$

for all $t, t^{\prime} \in T$. Furthermore we get:

$$
\begin{aligned}
p_{1} \varphi(t) & =p_{1}(\alpha(t), \beta(t)) \\
& =\alpha(t)
\end{aligned}
$$

and

$$
\begin{aligned}
p_{2} \varphi(t) & =p_{2}(\alpha(t), \beta(t)) \\
& =\beta(t)
\end{aligned}
$$

for all $t \in T$ that proves the commutativity of (1).

Consider $\varphi^{\prime}$ with the same property as $\varphi$, i.e. the following conditions hold:

$$
\begin{aligned}
& p_{1} \varphi^{\prime}=\alpha \\
& p_{2} \varphi^{\prime}=\beta .
\end{aligned}
$$

Define $(p, r) \in P \times R$ by $\varphi^{\prime}(t)=(p, r)$. We get:

$$
\begin{aligned}
p_{1} \varphi^{\prime}(t)=\alpha(t) & \Rightarrow p_{1}(p, r)=\alpha(t) \\
& \Rightarrow p=\alpha(t)
\end{aligned}
$$

and

$$
\begin{aligned}
p_{2} \varphi^{\prime}(t)=\beta(t) & \Rightarrow p_{2}(p, r)=\beta(t) \\
& \Rightarrow r=\beta(t)
\end{aligned}
$$


for all $t \in T$ which yields:

$$
\begin{aligned}
\varphi^{\prime}(t) & =(p, r) \\
& =(\alpha(t), \beta(t)) \\
& =\varphi(t)
\end{aligned}
$$

and proves that $\varphi$ is unique.

Theorem 2. The category of racks has pullbacks.

Proof. Let $f: P \rightarrow T$ and $g: R \rightarrow T$ be two rack morphisms. Define:

$$
P \times_{T} R=\{(p, r) \mid f(p)=g(r)\}
$$

which is a subrack of $P \times R$; see [5]. Then we get the following commutative diagram:

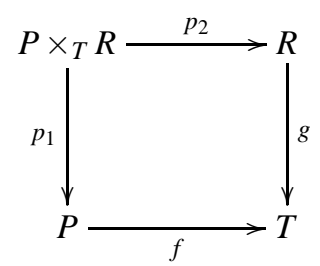

Let $Q$ be any rack with two rack morphisms $\alpha: Q \rightarrow P$ and $\beta: Q \rightarrow R$ where the following diagram commutes:

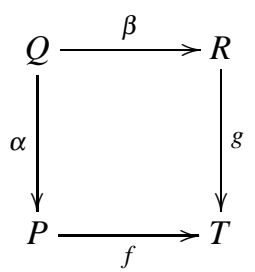

Then there must be a unique rack morphism:

$$
\varphi: Q \rightarrow P \times_{T} R
$$

that makes the following diagram commutative:

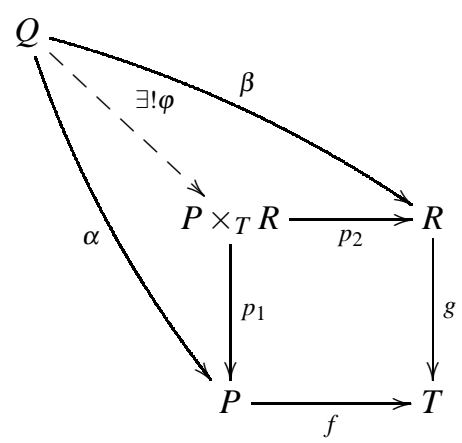


namely:

$$
\begin{aligned}
& p_{1} \varphi=\alpha \\
& p_{2} \varphi=\beta .
\end{aligned}
$$

For this aim, define:

$$
\begin{aligned}
\varphi: Q & \rightarrow P \times_{T} R \\
q & \mapsto \varphi(q)=(\alpha(q), \beta(q)) .
\end{aligned}
$$

Then $\varphi$ is a rack morphism since:

$$
\begin{aligned}
\varphi\left(q \triangleleft q^{\prime}\right) & =\left(\alpha\left(q \triangleleft q^{\prime}\right), \beta\left(q \triangleleft q^{\prime}\right)\right) \\
& =\left(\alpha(q) \triangleleft \alpha\left(q^{\prime}\right), \beta(q) \triangleleft \beta\left(q^{\prime}\right)\right) \\
& =(\alpha(q), \beta(q)) \triangleleft\left(\alpha\left(q^{\prime}\right), \beta\left(q^{\prime}\right)\right) \\
& =\varphi(q) \triangleleft \varphi\left(q^{\prime}\right)
\end{aligned}
$$

for all $q, q^{\prime} \in Q$. Furthermore we get:

$$
\begin{aligned}
p_{1} \varphi(q) & =p_{1}(\alpha(q), \beta(q)) \\
& =\alpha(q) \\
p_{2} \varphi(q) & =p_{2}(\alpha(q), \beta(q)) \\
& =\beta(q)
\end{aligned}
$$

for all $q \in Q$ that proves the commutativity of (2).

Consider $\varphi^{\prime}$ with the same property as $\varphi$, i.e. the following conditions hold:

$$
\begin{aligned}
& p_{1} \varphi^{\prime}=\alpha \\
& p_{2} \varphi^{\prime}=\beta .
\end{aligned}
$$

Define $(p, r) \in P \times_{T} R$ by $\varphi^{\prime}(q)=(p, r)$. We get:

$$
\begin{aligned}
p_{1} \varphi^{\prime}(q)=\alpha(q) & \Rightarrow p_{1}(p, r)=\alpha(q) \\
& \Rightarrow p=\alpha(q) \\
p_{2} \varphi^{\prime}(q)=\beta(q) & \Rightarrow p_{2}(p, r)=\beta(q) \\
& \Rightarrow r=\beta(q)
\end{aligned}
$$

for all $q \in Q$ which yields:

$$
\begin{aligned}
\varphi^{\prime}(q) & =(p, r) \\
& =(\alpha(q), \beta(q)) \\
& =\varphi(q)
\end{aligned}
$$

and proves that $\varphi$ is unique.

Theorem 3. The category of racks has equalizers.

Proof. Let $f, g: P \rightarrow R$ be two rack morphisms. Define the set:

$$
Q=\{p \in P \mid f(p)=g(p)\} .
$$


$Q$ is a subrack of $P$ since:

$$
\begin{aligned}
f\left(p \triangleleft p^{\prime}\right) & =f(p) \triangleleft f\left(p^{\prime}\right) \\
& =g(p) \triangleleft g\left(p^{\prime}\right) \\
& =g\left(p \triangleleft p^{\prime}\right)
\end{aligned}
$$

for all $p, p^{\prime} \in P$.

Also the inclusion morphism $u: Q \rightarrow P$ is a rack morphism since:

$$
\begin{aligned}
u\left(p \triangleleft p^{\prime}\right) & =p \triangleleft p^{\prime} \\
& =u(p) \triangleleft u\left(p^{\prime}\right)
\end{aligned}
$$

for all $p, p^{\prime} \in Q$. Furthermore for all $p \in Q$, we have:

$$
\begin{aligned}
(f u)(p) & =f(p) \\
& =g(p) \\
& =(g u)(p)
\end{aligned}
$$

and get:

$$
f u=g u .
$$

Let $T$ be any rack with a rack morphism $v: T \rightarrow P$ where:

$$
f v=g v .
$$

Then there must be a unique rack morphism:

$$
\phi: T \rightarrow Q
$$

such that the following diagram commutes:

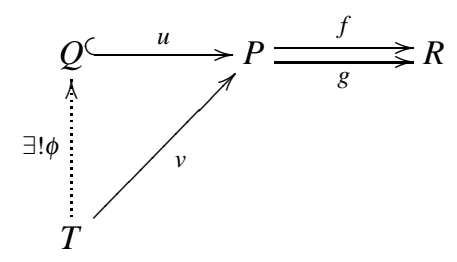

We can say that $v(t) \in Q$ since:

$$
f(v(t))=g(v(t))
$$

for all $t \in T$. Define $\phi$ by $\phi(t)=v(t)$ for all $t \in T$. Then we get:

$$
\begin{aligned}
u \phi(t) & =u v(t) \\
& =v(t)
\end{aligned}
$$

for all $t \in T$ that satisfies $u \phi=v$ and proves the commutativity of (3). 
Consider $\phi^{\prime}$ with the same property as $\phi$, i.e. $u \phi^{\prime}=v$. Define $q \in Q$ by $\phi^{\prime}(t)=q$. We get:

$$
\begin{aligned}
u \phi^{\prime}(t)=v(t) & \Rightarrow u(q)=v(t) \\
& \Rightarrow q=v(t)
\end{aligned}
$$

for all $t \in T$ which yields:

$$
\begin{aligned}
\phi^{\prime}(t) & =q \\
& =v(t) \\
& =\phi(t)
\end{aligned}
$$

and proves that $\varphi$ is unique.

\section{Acknowledgment}

The first author is partially supported by TÜBITAK.

\section{Competing interests}

The authors declare that they have no competing interests.

\section{Authors' contributions}

All authors have contributed to all parts of the article. All authors read and approved the final manuscript.

\section{References}

[1] Barr, M. and Wells, C., Category Theory, Lecture Notes for ESSLLI, (1999).

[2] Brieskorn, E., Automorphic Sets and Singularities, In "Braids (Santa Cruz, CA, 1986)”, Contemporary Mathematics, (1988), 78, p.45-115.

[3] Conway, J.H., Wraith, G., Unpublished Correspondence, (1959).

[4] Crans , A., Wagemann, F., Crossed modules of racks, Homology, Homotopy and its Appl., (2014), 16(2), p.85-106.

[5] Emir, K., Gülsün Akay, H., Pullback Crossed Modules in the Category of Racks, Doi: 10.15672/HJMS.2017.532.

[6] Emir, K., Çetin, S., Limits in Modified Categories of Interest, to appear in Bulletin of the Iranian Mathematical Society.

[7] Fenn, R., Rourke, C., Racks and Links in Codimension Two, J. Knot Theory Ramifications 1, ( 1992), p.343-406.

[8] Kauffman, L.H., Knot-Crystals Classical Knot Theory in Modern Guise, Knots and Physics, World Sci.

[9] Mac Lane, S., Categories for the working mathematician, Second edition, Graduate Texts in Mathematics, 5. Springer-Verlag, New York, (1998).

[10] Stanovský, D., Left distributive left quasigroup, Ph.D. Thesis, Charles University in prague, (2004). 